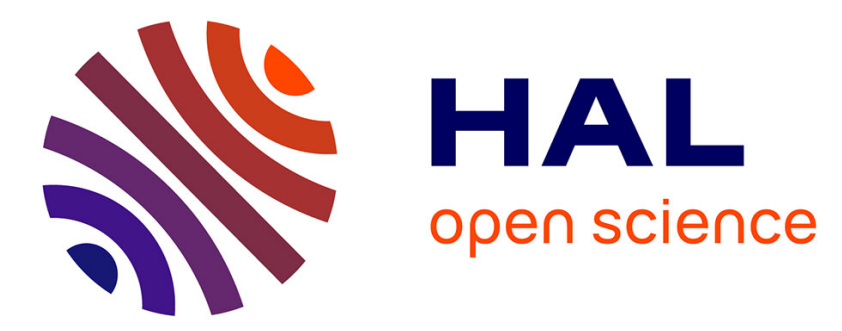

\title{
Random phase mask in a filamentation regime: application to the localization of point sources
}

Florence de La Barrière, Guillaume Druart, Nicolas Guérineau, Yann Ferrec, Jean Albert Taboury, Jérôme Primot

\section{- To cite this version:}

Florence de La Barrière, Guillaume Druart, Nicolas Guérineau, Yann Ferrec, Jean Albert Taboury, et al.. Random phase mask in a filamentation regime: application to the localization of point sources. Optics Letters, 2012, 36 (5), pp.684-686. 10.1364/OL.36.000684 . hal-00569371

\section{HAL Id: hal-00569371 https://hal.science/hal-00569371}

Submitted on 24 Feb 2011

HAL is a multi-disciplinary open access archive for the deposit and dissemination of scientific research documents, whether they are published or not. The documents may come from teaching and research institutions in France or abroad, or from public or private research centers.
L'archive ouverte pluridisciplinaire HAL, est destinée au dépôt et à la diffusion de documents scientifiques de niveau recherche, publiés ou non, émanant des établissements d'enseignement et de recherche français ou étrangers, des laboratoires publics ou privés. 


\title{
Random phase mask in a filamentation regime: application to the localization of point sources
}

\author{
Florence de la Barrière,,${ }^{1, *}$ Guillaume Druart, ${ }^{1}$ Nicolas Guérineau, ${ }^{1}$ Yann Ferrec, ${ }^{1}$ Jean Taboury, ${ }^{2}$ \\ and Jérôme Primot ${ }^{1}$ \\ ${ }^{1}$ ONERA, Chemin de la Hunière, 91761 Palaiseau cedex, France \\ ${ }^{2}$ Institut d'Optique, Campus Polytechnique RD 128, 91127 Palaiseau cedex, France \\ *Corresponding author: florence.de_la_barriere@onera.fr \\ Compiled December 14, 2010
}

\begin{abstract}
We present a new optical system with an extended point spread function (PSF) for the localization of point sources in the visible and infrared spectral ranges with a subpixel precision. This compact system involves a random phase mask (RPM) as its unique component. It exhibits original properties, since this RPM is used in a particular regime, called "filamentation regime", before the speckle region. The localization is performed by calculating the phase correlation between the PSF and the image obtained under off-axis illumination. Numerical simulations are presented to assess the basic optical properties of this RPM in the filamentation regime. (C) 2010 Optical Society of America

OCIS codes: 030.5770, 030.6600, 070.7345.
\end{abstract}

The localization of point sources is an important issue, which has been widely addressed in literature. It has been shown that an extended optical point spread function (PSF) is of particular interest for the localization of point sources which are separated by a subpixel distance with a great precision [1]. Optical systems with an extended PSF have already been described. Some are based on coded apertures, which were originally used for imagery in the fields of X-rays and gamma rays [2], such as random arrays of pinholes [3]. Due to the issues which arise when it comes to adapting coded apertures to the visible and infrared spectral ranges [4], alternatives have been proposed: in [1], a pseudorandom phase mask is associated with a lens, resulting in an extended PSF of the overall system. This principle is emphasized in [5], which describes a multichannel system based on the TOMBO principle [6], where the PSF of each channel is extended thanks to an array of Zernike phase masks. In this Letter, we propose removing the lens in the system described in [1]. Then the random phase mask (RPM) is used as the unique component. This approach follows a general trend which consists in designing optical systems with a minimal number of elements (an aperture stop, an optical component and a detector) [7]. Moreover, we propose making use of the RPM in an original regime, called "filamentation regime", before the speckle region. Based on simulations, this Letter aims at showing that the RPM used in the filamentation regime has an extended PSF which enables the localization of point sources with a subpixel precision.

The RPM which is considered in this study is a thin plate with an index of refraction $n$. One face of the plate is flat, and the other face has a stationary Gaussian random surface roughness. The height difference between the mean flat surface and the random surface at point $\mathbf{r}$ of the surface is denoted $h(\mathbf{r})$ (cf. Fig. 1(a)). The probability density function of the roughness of the surface $h(\mathbf{r})$ is Gaussian, and expressed in the following way:

$$
P_{h}(h)=\frac{1}{\sqrt{2 \Pi \sigma_{h}^{2}}} \exp \left(-\frac{h^{2}}{2 \sigma_{h}^{2}}\right),
$$

where $\sigma_{h}$ stands for the standard deviation of the random variable $h$.

The covariance of $h$ is radial, and is given by:

$$
\operatorname{Cov}_{h h}(\mathbf{r})=\sigma_{h}^{2} \exp \left(-\frac{r^{2}}{2 R_{c}^{2}}\right) .
$$

The RPM is thus described by three parameters: its index of refraction $n$, the standard deviation $\sigma_{h}$ of the roughness $h$, and the size of the spatial pattern of the phase mask $R_{c}$. We assume that the phase introduced by the mask has smooth spatial variations, which means that $R_{c}>>\sigma_{h}>>\lambda$, where $\lambda$ is the wavelength. If we consider that the mask is thin, the transmittance $t(\mathbf{r})$ at point $\mathbf{r}$ of the component is given by :

$$
t(\mathbf{r})=\exp \left(j \frac{2 \Pi}{\lambda}(n-1) h(\mathbf{r})\right) \operatorname{disc}\left(\frac{\mathbf{r}}{a}\right),
$$

where $a$ is the radius of the pupil, and disc is the function defined as: $\operatorname{disc}(\mathbf{r})=1$ if $\|\mathbf{r}\| \leq 1$, and $\operatorname{disc}(\mathbf{r})=0$ otherwise.

To calculate the PSF of the RPM, the component is illuminated by a monochromatic plane wave parallel to its optical axis $(\lambda=500 \mathrm{~nm})$. Using the formalism of Fresnel wave propagation in free space, the intensity distribution is calculated in transversal planes beyond the RPM along the $z$-axis. We note $d$ the distance between the exit plane of the RPM and the observation plane (cf. Fig. 1(a)). While $d$ increases, we first observe the formation of filaments in the image, followed by the speckle regime. The distance for which the filaments are the thinnest is considered to be the optimal working distance $d_{\text {opt }}$ of the RPM, because the energy is concentrated in 
specific zones of the image. This corresponds to an original regime which we call "filamentation regime". Since our goal is to obtain a very textured image, we define a signal-to-noise ratio by the following equation:

$$
S N R=\frac{\sqrt{\operatorname{Var}(I)}}{E[I]},
$$

where $I$ is the intensity in the observation plane, $\operatorname{Var}(I)$ and $E[I]$ are respectively the variance and the mean value of $I$ over the geometric projection of the pupil on the image plane. Thin filaments lead to high $S N R$.

Fig. 1(b) shows the calculated $S N R$ under monochromatic illumination $(\lambda=500 \mathrm{~nm})$ as a function of the distance $d$. The optimal working distance $d_{\text {opt }}$ of the RPM corresponds to the value of $d$ for which the $S N R$ is maximum. When $d$ is greater than $\Pi a^{2} / \lambda$ (in the Fraunhofer diffraction regime), the curve tends towards 1 , which is the value of the $S N R$ of a fully developed speckle [8].

The optimal working distance $d_{o p t}$ can be interpreted in terms of conventional optical components. Indeed, as the shape of the mask is smooth with respect to the wavelength, the RPM can be seen as a collection of small toroïdal lenses, whose focal lengths are given by the local curvatures of the surface. Besides, from several simulations of the RPM (carried out by varying its definition parameters $n, R_{c}$ and $\sigma_{h}$ ), we have inferred an empirical formula for the optimal working distance of the RPM:

$$
d_{o p t}=\frac{1}{2(n-1)} \frac{R_{c}^{2}}{\sigma_{h}}
$$

We can notice that $d_{\text {opt }}$ is the focal length of a planoconvex lens of diameter $2 R_{c}$ and of on-axis thickness $\sigma_{h}$, when $R_{c}>>\sigma_{h}$.

If we do not take into account the variation of $n$ with $\lambda$, we can see that $d_{o p t}$ does not depend on the wavelength and thus assume that the filamentation regime is achromatic. The intensity distributions in transversal planes for two values of $d$ are shown in Figs. 1(c) (in the filamentation regime) and 1(d) (in the fully developed speckle regime). These illustrations are obtained with a RPM defined by the following parameters: $R_{c}=80 \mu \mathrm{m}$, $\sigma_{h}=3 \mu m$ and $n=1.45$. Figs. $1(\mathrm{c})$ and $1(\mathrm{~d})$ result of the superposition of monochromatic images obtained for three values of wavelengths: $\lambda_{\text {blue }}=400 \mathrm{~nm}$ (blue channel), $\lambda_{\text {green }}=500 \mathrm{~nm}$ (green channel) and $\lambda_{\text {red }}=600 \mathrm{~nm}$ (red channel). According to the theory related to speckle phenomena, the fully developed speckle regime is chromatic (because the size of the speckle pattern depends on $\lambda)$. However, as expected, the filaments are white in the filamentation regime, which means that their position in the image plane is achromatic (except a few irisations which we can see in Fig. 1(c)).

An optimal configuration for the localization of point sources is obtained when the size of the RPM is greater than the size of the detector [3]. The on-axis overall PSF of the RPM is illustrated in Fig. 2(a). Fig. 2(b) shows the portion of the on-axis PSF which is imaged on the
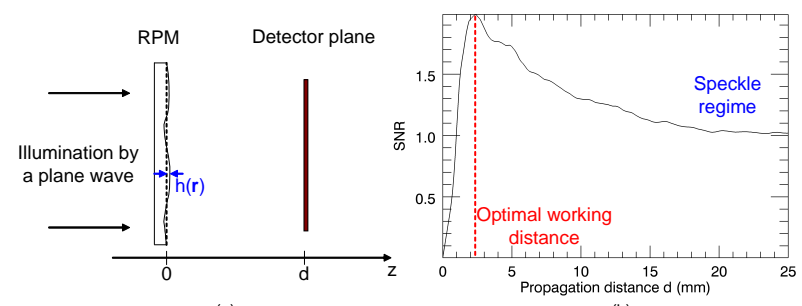

(a)

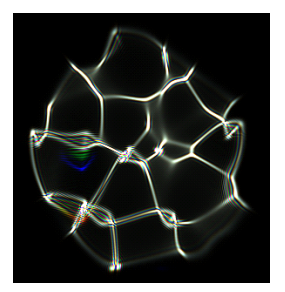

(c)

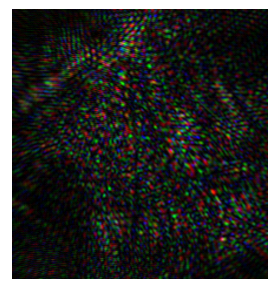

(d)

Fig. 1. (Color online) (a) Illumination of the RPM by a plane wave, (b) Calculated $S N R$ under monochromatic illumination as a function of $d$, (c) Polychromatic intensity distribution at the optimal working distance, (d) Polychromatic intensity distribution in speckle regime.

detector. We now focus on the off-axis behaviour of the RPM: the component is illuminated by a monochromatic plane wave which is tilted with an angle equal to $10^{\circ}$ with respect to the optical axis; the intensity pattern is shown in Fig. 2(c). Fig. 2(d) illustrates the portion of this off-axis pattern imaged on the detector. The phase correlation [9] of the images illustrated in Figs. 2(d) and 2(a) exhibits a two-dimensional very thin spot. The profile of this thin spot along one axis is shown in Fig. 3. Then, the barycentre of this thin spot gives the position of the point source.

The correlation peak can be used to define the angular resolution $I F O V$ of the system: $I F O V=F W H M / d_{o p t}$, where $F W H M$ is the full width at half maximum of the phase correlation peak (cf. Fig. 3). For this simulation, we find a $F W H M$ equal to $8.8 \mu \mathrm{m}$ for $d_{o p t}=2.4 \mathrm{~mm}$, which leads to $I F O V=0.2^{\circ}$. It is worth mentioning that in this Letter, the definition parameters of the RPM have not been optimized to decrease IFOV: IFOV can then be chosen for a given application by properly adjusting the RPM parameters.

To conclude, we have shown that a RPM used as the unique component in the filamentation regime generates an extended PSF, which enables to perform the localization of point sources with a subpixel precision. It exhibits several advantages. One of these is that it can be very compact, provided a suitable choice of its parameters $n$, $R_{c}$ and $\sigma_{h}$. Moreover, we can notice that the on- and off-axis images (Figs. 2(a) and 2(c)) are very similar, except from the global translation of the intensity distribution in the observation transverse plane. Therefore, the RPM suffers from a low amount of off-axis aberrations; then, we could expect that this component will have a great field of view. Within future work, we are 

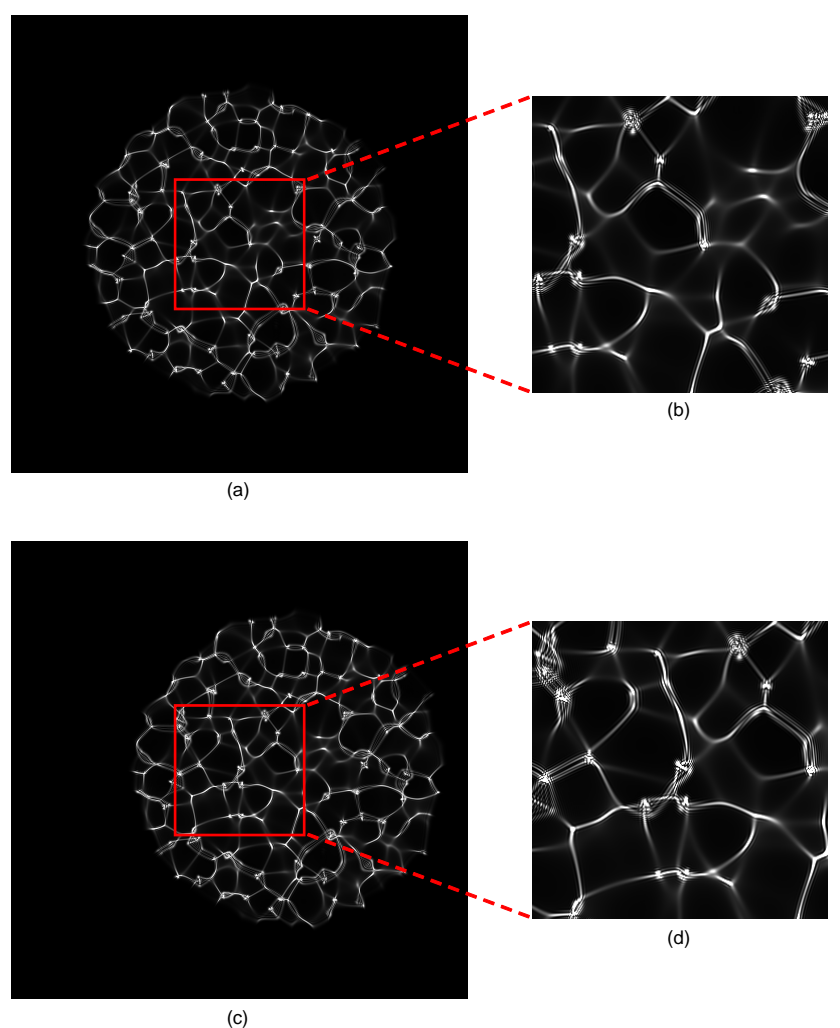

Fig. 2. (Color online) (a) On-axis monochromatic PSF of the RPM, the red square stands for the position and the size of the detector, (b) Portion of the on-axis PSF imaged on the detector, (c) Off-axis monochromatic illumination of the RPM (angle of incidence of the plane wave: $10^{\circ}$ ), (d) Portion of (c) imaged on the detector.

going to address the optimization of the RPM parameters, and to evaluate the localization performance of the RPM (and especially study the slight distortion of the PSF with the field angle and the phase correlation under polychromatic illumination).

This work was sponsored by the Délégation Générale pour l'Armement (DGA) of the French Ministry of Defense. It results from the PRECISION collaboration between ONERA and Institut d'Optique.

\section{References}

1. A. Ashok, M. A. Neifeld, "Pseudorandom phase masks for superresolution imaging from subpixel shifting," Appl. Opt. 46, 2256 (2007).

2. R. H. Dicke, "Scatter-hole cameras for X-rays and gamma rays", Astrophys. J. 153, p. L101 (1968).

3. E. E. Fenimore, T. M. Cannon, "Coded aperture imaging with uniformly redundant arrays", Appl. Opt. 17, 337 (1978).

4. S. R. Gottesman, A. Isser, G. W. Gigioli, "Adaptative coded apertures: bridging the gap between nondiffractive and diffractive imaging systems", Proc. SPIE 7818, 781805-1 (2010).

5. A. Ashok, M. A. Neifeld, "Point spread function engineering for iris recognition system design," Appl. Opt.

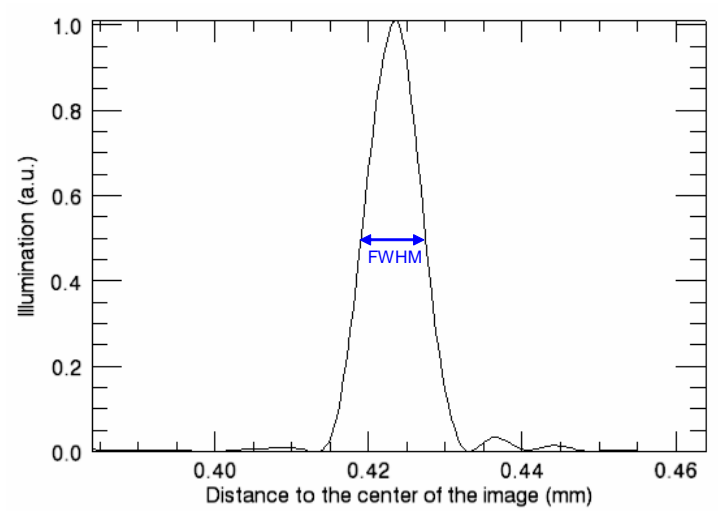

Fig. 3. (Color online) Line profile centered on the correlation peak along one direction of the phase correlation between Figs. 2(d) and 2(a).

49, B26 (2010).

6. J. Tanida, T. Kumagai, K. Yamada, S. Miyatake, K. Ishida, T. Morimoto, N. Kondou, D. Miyazaki, Y. Ichioka, "Thin Observation Module by Bound Optics (TOMBO): Concept and Experimental Verification," Appl. Opt. 40, 1806 (2001).

7. F. de la Barrière, G. Druart, N. Guérineau, J. Taboury, "Design strategies to simplify and miniaturize imaging systems," Appl. Opt., doc. ID 135346 (accepted 09 November 2010, in press).

8. J. W. Goodman, Speckle phenomena in optics: theory and applications, (Roberts and Company Publishers, 2007), Chapter 3, p. 29.

9. Ph. Refregier, "Optimal trade-off filters for noise robustness, sharpness of the correlation peak, and Horner efficiency," Opt. Lett. 16, 829 (1991). 


\section{Informational Fourth Page}

In this section, full versions of citations to assist reviewers and editors (OL publishes a short form of citations) are provided.

\section{References}

1. A. Ashok, M. A. Neifeld, "Pseudorandom phase masks for superresolution imaging from subpixel shifting," Appl. Opt. 46, 2256-2268 (2007).

2. R. H. Dicke, "Scatter-hole cameras for X-rays and gamma rays", Astrophys. J. 153, p. L101 (1968).

3. E. E. Fenimore, T. M. Cannon, "Coded aperture imaging with uniformly redundant arrays", Appl. Opt. 17, 337-347 (1978).

4. S. R. Gottesman, A. Isser, G. W. Gigioli, "Adaptative coded apertures: bridging the gap between nondiffractive and diffractive imaging systems", Proc. SPIE 7818, 781805-1-781805-9 (2010).

5. A. Ashok, M. A. Neifeld, "Point spread function engineering for iris recognition system design," Appl. Opt. 49, B26-B39 (2010).

6. J. Tanida, T. Kumagai, K. Yamada, S. Miyatake, K. Ishida, T. Morimoto, N. Kondou, D. Miyazaki, Y. Ichioka, "Thin Observation Module by Bound Optics (TOMBO): Concept and Experimental Verification," Appl. Opt. 40, 1806-1813 (2001).

7. F. de la Barrière, G. Druart, N. Guérineau, J. Taboury, "Design strategies to simplify and miniaturize imaging systems," Appl. Opt., doc. ID 135346 (accepted 09 November 2010, in press).

8. J. W. Goodman, Speckle phenomena in optics: theory and applications, (Roberts and Company Publishers, 2007), Chapter 3, p. 29.

9. $\mathrm{Ph}$. Refregier, "Optimal trade-off filters for noise robustness, sharpness of the correlation peak, and Horner efficiency," Opt. Lett. 16, 829-831 (1991). 